$2853 \mathrm{~cm}-1$, as biomarkers of apoptosis, were assessed.

Our in vitro biological screening system detected embryotoxic/anti-tumor impact of both HDIs. FTIR spectroscopy was able to discern biomarkers of histone acetylation and apoptosis in spent media metabolomes, thus upgrading our biological in vitro system for faster screening of embryotoxic/antitumor agents.

\section{RARE COPY NUMBER VARIANTS IN CONGENITAL HEART DEFECTS}

Maša Davidović*, Leona Morožin Pohovski, Nikolina Vidan Rogulj, Ivona Sansovic, Adriana Bobinec, Ana-Maria Meašić, Mijana Kero, Ljubica Boban, Ivan Malčić, Ingeborg Barišic. Department of Paediatrics, University Hospital Centre Zagreb, University of Zagreb School of Medicine, Zagreb, Croatia

\subsection{6/archdischild-2021-europaediatrics.84}

To detect copy number variants (CNVs) in patients with congenital heart defects (CHD) and identify potential novel candidate genes involved in $\mathrm{CHD}$ pathogenesis. $\mathrm{CHD}$ are the most common congenital anomalies. Etiology of CHD can be genetic (chromosomal abnormalities, rare single gene disorders) or environmental, but it is mostly multifactorial. Copy number variants (CNVs) are important causes of genetic syndromes associated with CHD. Chromosomal microarray (CMA) is used as the first test to detect the CNVs in this category of patients.

We have evaluated 260 subjects with CHD in the period between Jan 2016 and Sep 2019 using CMA. In the majority of cases additional features such as facial dysmorphia, intellectual disability/developmental delays (ID/DD) or extracardiac anomalies (ECA) $(190 / 260 ; 73.1 \%)$ were present; the remainder had isolated CHD. CMA analysis was performed using Agilent SurePrint G3 Unrestricted CGH ISCA v2 Human Genome, 8x60 K oligonucleotide microarray format according to the manufacturers' instructions (Agilent Technologies, USA).

Copy number variants were detected in 71 (71/260, $27.3 \%)$ patients; 53 of them $(53 / 71,74.6 \%)$ were classified as pathogenic and $18(18 / 71,25.4 \%)$ as variants of unknown clinical significance (VUS). Pathogenic CNVs were predominately deletions (36), followed by duplications (9) and complex rearrangements (8). CNVs were discovered in 4 patients with isolated CHD $(4 / 70,5.7 \%)$ and 67 patients with additional feature/s (67/190; 35.3\%). The most frequent pathogenic CNV was 22q11.21 deletion (DiGeorge syndrome), followed by other well-known syndromes (Williams, 5p deletion etc.). Beside these, we detected CNV clusters in loci that have previously been associated with CHD in literature (such as $15 \mathrm{q} 11.2,8 \mathrm{p} 23.1,1 \mathrm{q} 43$ etc.). We also observed rare CNVs in loci that have not yet been recognized as important in CHD pathogenesis (i.e. Yp11.2, 17q24, 15q24.3 etc.) Identification of rare CNVs is important for clarification of $\mathrm{CHD}$ pathogenesis. Potential novel candidate genes in these loci warrant further research.

This work was supported by Scientific Center of Excellence for Reproductive and Regenerative Medicine and by the European Union through the European Regional Development Fund, under grant agreement No. KK.01.1.1.01.0008, project „Reproductive and Regenerative Medicine - Exploring New Platforms and Potentials.

\section{CLINICAL, CYTOGENETIC AND MOLECULAR FINDINGS IN PATIENTS WITH PALLISTER-KILLIAN SYNDROME}

Ivana Milković*, Ivana Tonković Đurišević, Kristina Crkvenac Gornik, Anita Pokupec Bilić, Marija Vidaković, Sanda Huljev Frković. University Hospital Centre Zagreb, Department of Pediatrics

10.1136/archdischild-2021-europaediatrics.85

Goal Pallister Killian syndrome is a rare genetic disorder caused by tissue-limited mosaicism tetrasomy of the short arm of chromosome 12, which usually presents as an extra isochromosome 12p. Clinical features include distinct facial anomalies, other systemic abnormalities with variable developmental delay and intellectual impairment. The aim of this work is to present clinical and cytogenetic findings of PKS patients diagnosed in our Clinic for the last 12 years and to compare their findings with previously published cases.

Methods The suspicion of PKS was set after the recognition of their characteristic phenotypic features. The diagnosis was confirmed by karyotype analysis of fibroblast cultures and In situ hybridization with chromosome 12-specific DNA, which revealed the supernumerary mosaic $\mathrm{i}(12 \mathrm{p})$.

Results Since 2008 four patients with PKS were diagnosed and treated at our Clinic. Karyotypes obtained from cultured peripheral lymphocytes were normal in two cases, while karyotypes obtained from cultured skin samples revealed the supernumerary mosaic $\mathrm{i}(12 \mathrm{p})$ in all four patients.

Conclusion The wide phenotypic spectrum of PKS in conjunction with the mosaic distribution of the $i(12 p)$ makes PKS often an underdiagnosed disorder. Since additional chromosome is usually absent in karyotypes obtained from cultured peripheral lymphocytes, clinical recognition with skin biopsy and fibroblast chromosome examination is of utmost importance.

\section{FLOPPY INFANT SYNDROME DUE TO CONNECTIVE TISSUE DISORDER. CASE REPORT OF A PATIENT WITH KYPHOSCOLIOTIC EHLERS-DANLOS SYNDROME}

Tomislav Smoljo*, Sanda Huljev Frković, Nina Barišić, Ivo Barić, Danijela Petković Ramadža. University of Zagreb, School of Medicine

\subsection{6/archdischild-2021-europaediatrics.86}

Introduction Ehlers-Danlos syndrome is a group of 13 hereditary connective tissue disorders due to defects in collagen formation, folding or interaction. Kyphoscoliotic EDS (kEDS) is characterized by severe congenital hypotonia, early-onset progressive kyphoscoliosis, joint hypermobility, hyperelastic skin and myopathy. The majority of patients have biallelic mutations in the PLOD1 gene, and others in the FKBP14 gene. The clinical phenotype is indistinguishable, except for hearing impairment, occurring in FKBP14-kEDS only. FKBP14-kEDS (OMIM 614557) is an ultra-rare disease with less than 30 patients described. We present our patient in order to raise awareness of connective tissue disorders in the differential diagnosis of floppy infant syndrome.

Case Presentation The patient was born after a normal pregnancy and delivery. Family history is unremarkable. Parents are unrelated. Joint hypermobility and weak cry were present at birth. Hearing screening revealed left-sided hearing impairment, confirmed by audiometry. Brain, heart, abdominal ultrasound and ophthalmic examination were normal. After the 